\title{
Valoración del patrimonio geológico en Europa
}

\author{
Valuation of geoheritage in Europe
}

\author{
LUIS CARCAVILLA ${ }^{1}$, ENRIQUE DÍAZ-MARTÍNEZ ${ }^{1}$, LARS ERIKSTAD² ${ }^{2}$ ÁNGEL GARCÍA-CORTÉS ${ }^{1}$. \\ IInstituto Geológico y Minero de España (IGME) - I.carcavilla@igme.es - e.diez@igme.es - garcia.cortes@igme.es \\ ${ }^{2}$ Norwegian Institute for Nature Research (NINA) - lars.erikstad@nina.no
}

\begin{abstract}
Resumen
Los orígenes del estudio del patrimonio geológico en Europa se sitúan en la segunda mitad del siglo XX, si bien mucho antes ya se habían puesto en marcha iniciativas para la protección de enclaves singulares de interés estético. Desde entonces, cada una de las 50 naciones europeas ha diseñado distintas estrategias y mecanismos para el estudio y conservación del patrimonio geológico, con diferente grado de desarrollo y avance. Este ha dependido generalmente de la iniciativa de los geocientíficos pero también del interés mostrado por las administraciones competentes en conservación del patrimonio natural, de manera que, aunque cada país tiene su problemática particular, hay muchas situaciones comunes. Algunos programas globales promovidos desde Europa, como la Red Global de Geoparques y Global Geosites, han impulsado el inventario y divulgación del patrimonio geológico, si bien a día de hoy no existe una directiva europea que promueva la conservación del patrimonio geológico, lo que sin duda impulsaría definitivamente la geoconservación en todos lo estados. Algunos países pueden servir de ejemplo al destacar en la elaboración de inventarios nacionales, en la legislación o en el diseño de metodologías para la geoconservación, pero en términos generales, el patrimonio geológico en Europa siempre ha ido a remolque de las iniciativas de conservación de la biodiversidad. Aún queda mucho camino por recorrer para desarrollar sistemas de valoración y estrategias de geoconservación en todos los países.
\end{abstract}

Palabras clave: Europa; geodiversidad; geoconservación; patrimonio geológico.

\begin{abstract}
Although the origins of geoheritage studies in Europe date back to the second half of the 20th century, many initiatives had been implemented long time before but towards the protection of unique sites with aesthetic interest. Since then, each of the 50 European nations has designed and implemented different strategies and methodologies towards the study and conservation of geoheritage, but with different degrees of development and advance. This has generally relied on the geoscientists initiative, but also the interest shown by public administrations in the conservation of natural heritage, so that, even if each country has its own specific problems, there are also many common situations. Some global programs promoted from Europe, such as the Global Geopark Network and Global Geosites, have promoted inventories and public outreach on geoheritage. Nowadays, there is not a common European directive promoting the conservation of geoheritage, which would certainly promote geoconservation. Some countries may serve as examples of the implementation of national inventories, legislation or design of methodologies towards geoconservation, but in general terms, geoheritage in Europe has always gone behind biodiversity conservation initiatives. There is still much to be done in order to develop geoheritage assessment systems (valuing) and geoconservation strategies in all countries.
\end{abstract}

Key words: Europe; geoconservation; geodiversity; geoheritage.

\section{INTRODUCCIÓN}

Los orígenes del estudio del patrimonio geológico en Europa se sitúan en la segunda mitad del siglo XX, cuando tuvieron lugar diversas iniciativas puntuales, generalmente basadas en experiencias concretas de geoconservación en afloramientos emblemáticos realizadas incluso décadas antes. En cada una de las 50 naciones europeas se han diseñado distintas estrategias y mecanismos para el estudio y conservación del patrimonio geológico, con diferente grado de desarrollo y avance en función del interés mostrado por las administraciones competentes y de los recursos disponibles. Desde el continente europeo se han promovido diversas declaraciones, programas $y$ recomendaciones con el ánimo de impulsar la conservación del patrimonio geológico y, aunque cada país tiene su problemática particular, sí hay muchas situaciones comunes. Algunos programas, como el de la Red Global de Geoparques (GGN, de sus siglas en inglés), sirven de impulso para el inventario, conservación y divulgación del patrimonio geológico, pero es a escala nacional donde más énfasis debería hacerse, ya que actualmente no existe una directiva europea que fomente la conservación del patrimonio geológico, como sí existe para la biodiversidad. Así que, en términos generales, y a pesar de lo mucho que se ha avanzado en esta materia, se puede seguir afirmando que la conservación del patrimonio geológico en Europa no está generalizada y que, a pesar de los importantes avances en las últimas décadas, aún queda mucho camino por 
recorrer para implantar sistemas eficientes de valoración y de geoconservación.

\section{ORÍGENES DEL ESTUDIO DEL PATRIMONIO GEOLÓGICO Y LA GEOCONSERVACIÓN EN EUROPA}

El estudio del patrimonio geológico figura entre las más recientes áreas de investigación incorporadas al ámbito de la Geología. Es el resultado de una nueva manera de entender los recursos geológicos como bienes con interés científico y cultural que deben ser protegidos y que pueden ofrecer interesantes posibilidades para el desarrollo local. Por ello, el estudio del patrimonio geológico es cada vez más multidisciplinar, incorporando en su gestión aspectos relacionados con la legislación, los espacios protegidos, el turismo o la economía. El objetivo final del estudio del patrimonio geológico es promover su conservación, más aún teniendo en cuenta que la mayoría de los enclaves de alto valor geológico son recursos no-renovables, por lo que su destrucción es irreversible. Por ello, desde hace unos años se habla de geoconservación, en referencia a las técnicas de conservación del patrimonio geológico, que a menudo difieren de las utilizadas en otros aspectos del medio natural.

A pesar de los importantes avances realizados en Europa en las últimas décadas, todavía hay claras deficiencias en el desarrollo metodológico acerca del patrimonio geológico, su estudio sistemático, e incluso el reconocimiento general en el propio colectivo de estudiosos de las Ciencias de la Tierra. Se puede afirmar que el patrimonio geológico es el gran olvidado en la protección del medio ambiente en la actualidad. Incluso se lo sigue considerando como la "cenicienta" de las políticas de conservación de la naturaleza, pues es, a menudo, eclipsada por el interés suscitado por los aspectos bióticos del medio natural. A pesar de su innegable valor, es una constante en Europa que su protección ha ido siempre a remolque de los pasos dados en materia de conservación del medio biótico. Sin embargo, desde hace unas décadas, el panorama nacional e internacional está cambiando. Las principales entidades científicas geológicas se han ido haciendo eco de la necesidad de conservar el patrimonio geológico, e incluso han creado comisiones y grupos de trabajo ad hoc. Han surgido asociaciones específicas orientadas al estudio del patrimonio geológico, e incluso la atención al patrimonio geológico empieza a ser participativa y surge como iniciativa de entidades locales y de voluntarios. Y lo que es más importante, diversos países han impulsado los inventarios nacionales y han promulgado leyes específicas para la conservación del patrimonio geológico o con mención explícita dentro de leyes de patrimonio natural. El objetivo del presente trabajo es realizar un repaso a esta evolución y, sobre todo, a los conceptos y diversas interpretaciones sobre el patrimonio geológico.
El estado de los estudios, especialmente en materia de conservación, muestra un avance desigual según los países. Hay casos en los que existe tradición en el estudio del patrimonio geológico, y otros donde su avance es muy reciente o escaso. Desde el impulso nacional se ha pasado al diseño de planes internacionales, e incluso existen notables experiencias de acciones participativas de asociaciones no gubernamentales y de voluntariado, cuyos resultados en materia de conservación empiezan a ser patentes. En muchos casos, los términos hasta ahora definidos se integran en una serie de programas nacionales o internacionales de patrimonio geológico, siendo denominados por siglas referidas al proyecto en el que se inscriben. Se trata, por tanto, de conceptos análogos, pero que son denominados de diferente manera en función del marco legal, administrativo o conceptual en el que se inscriban.

Los trabajos sobre patrimonio geológico y geoconservación realizados en diversos países europeos dieron lugar a que en 1988 se reunieran geólogos de siete países (Austria, Dinamarca, Finlandia, Reino Unido, Irlanda, Noruega y Holanda) para poner en común sus ideas y problemáticas. Esta "primera reunión internacional de geoconservación" incluía entre sus temas fundamentales cómo afrontar el proceso de selección y clasificación del patrimonio geológico y su posterior gestión garantizando su conservación. Esta primera cita sirvió de base para que se realizaran varias reuniones más, entre las que destaca la que tuvo lugar en Digne (Francia) en 1991, y a la que acudieron más de un centenar de especialistas, incluyendo geólogos de otros países, como Suiza, Francia, España y Bélgica. Esta reunión se considera el germen de una verdadera conciencia geoconservacionista entre los geólogos europeos. Fruto del trabajo conjunto, surgió en 1991 la llamada Declaración de Digne (Martini, 1994), que establece las bases conceptuales y teórica en las que se basa la geoconservación. A pesar de que han pasado más de 20 años desde su redacción, sigue vigente y los principios en los que se inspira siguen siendo válidos. Uno de los efectos de esta reunión fue que tan sólo dos años más tarde, en 1993, se creó la Asociación Europea para la Conservación del Patrimonio Geológico (ProGEO), de la que hablaremos más adelante.

\section{ALGUNAS CARACTERÍSTICAS DE LA GEOCONSERVACIÓN EN EUROPA}

Como ya se ha comentado, aunque cada nación europea ha llevado su propio camino a la hora de inventariar, proteger y divulgar su patrimonio geológico, hay una serie de características comunes que se repiten de manera sistemática. Cabe destacar que, en muchos países europeos, los primeros pasos de la geoconservación son realmente antiguos. Generalmente se trata de la protección de algunos afloramientos 
geológicos singulares, gracias a la mediación de personajes de la nobleza y realeza de la época. Por ejemplo, uno de los antecedentes más antiguos es la emisión de un decreto con fines conservacionistas para controlar el acceso a la cueva Baumann (Alemania), emitido en 1668 por el Duque Rudolf August. También en Alemania, en 1832, se protegió el Drachenfels, cerca de Bonn, por iniciativa del Príncipe de la corona prusiana Friederich Wilhelm; en 1840, el rey de Bavaria promovió la protección de un corte en rocas jurásicas cerca de Weltenburg; y en 1844 fue protegida una roca granodirítica llamada Totentein en Sajonia (Röhling, 2012). Pero Alemania no es el único caso. Por ejemplo, en 1856, el conservador del Museo de Linz convenció a la

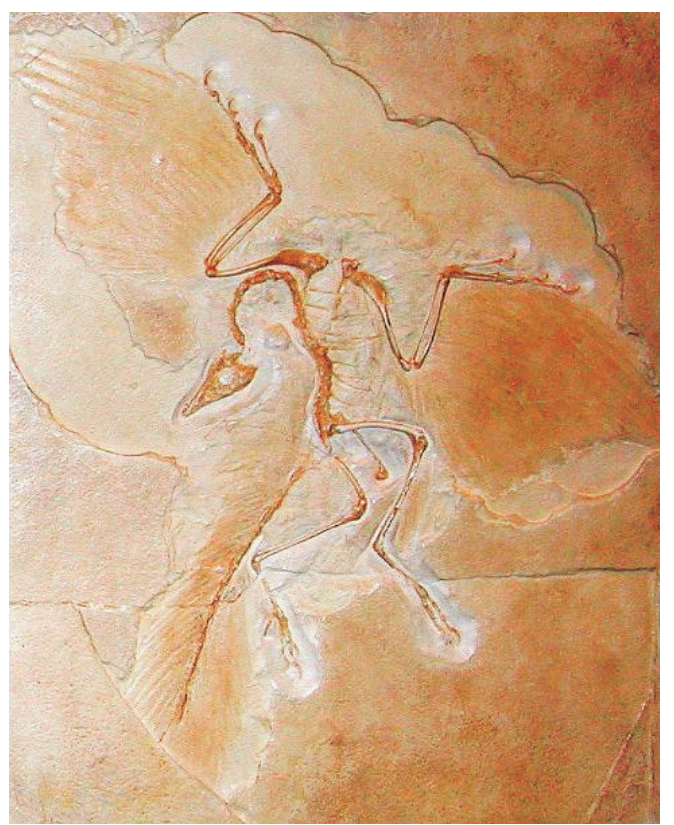

Figura 1 - Fósil de Archaeopteryx encontrado en las canteras de caliza litográfica de Solnhofen (Alemania), un KonservatLagerstätte del Jurásico. Los primeros fósiles de Archaeopteryx se encontraron en estas canteras a finales del siglo XIX.

Asociación Austriaca de Doctores y Científicos para que comprara un terreno con objeto de evitar que fueran explotados en cantera unos bloques exóticos de granito (Hofmann, 2012). También a mediados y finales del siglo XIX fueron encontrados interesantes ejemplares fósiles que formaron parte de colecciones de museos (fig.1). Así que parece que el origen de la preocupación por la conservación y valoración del patrimonio geológico surgió de la conciencia conservacionista iniciada en algunos países a finales del siglo XIX y principios del XX, que entendía que ciertos enclaves naturales poseen un notable valor por sí mismos y que, por lo tanto, deben ser protegidos y conservados.
Dentro de estos iniciales movimientos conservacionistas del medio natural, fue la protección de ciertos elementos geológicos la que adquirió mayor relevancia, debido a su alto valor escénico.

En relación con la protección de lugares amenazados, la declaración del primer parque nacional del mundo, el de Yellowstone en Estados Unidos en 1872, sin duda marcó un modelo de gestión basado en la declaración de áreas protegidas que tuvo reflejo en los países europeos. Así, Suecia fue el primer país europeo en declarar una ley de conservación de la naturaleza en 1909. Otros países se irían sumando posteriormente a este enfoque creando sus propias leyes de conservación de la naturaleza, como Noruega en 1910, Estonia en 1916 y España en 1917. Sin embargo, cabe destacar que, en 1837, el rey Leopold I de Bélgica ya había creado la Comisión Real de Monumentos y Paisajes con responsabilidad en la protección de monumentos naturales (Jacobs, 2012). Fruto de estas legislaciones conservacionistas del patrimonio natural, tuvo lugar una primera época de protección de lugares de interés geológico, generalmente enclaves paisajísticos emblemáticos. Así, en 1919 se protegió el fiordo de Oslo en Noruega y en 1923 varios bloques erráticos en el suroeste del país (Erikstad, 2012); en 1920 fueron protegidos en Holanda un afloramiento del Carbonífero y un acantilado costero sobre un till Ilamado Mirdunmmerklif (Ancker \& Jungerius, 2012); y en 1924 se declaró el Parque Natural Alpino del Mt. Triglav en Eslovenia (Hlad, 2012). Por su parte, Polonia estableció en 1926 una comisión de protección de la naturaleza que dos años más tarde publicó el listado de Monumento Naturales Inanimados de la República Polaca (Malkowski, 1928; Alexandrowicz, 2012), mientras que en España se declararon en 1918 sus dos primeros parques nacionales, ambos con una gran significación geológica, y en 1927 diversos Sitios Naturales de Interés Nacional mediante los cuales se protegieron enclaves de gran significación geológica (Carcavilla et al., 2009) (fig.2). Por tanto, son muchos los países europeos que durante las primeras tres décadas del siglo XX protegieron enclaves geológicos singulares, en el marco de una política de protección de la naturaleza con un cierto aire romántico, dando prioridad a los paisajes emblemáticos, en una mezcla de emotividad y admiración por la naturaleza, más que con un verdadero criterio científico o de representatividad. La Primera Guerra Mundial (1914-1917) supuso un paréntesis en esta tendencia pero no una ruptura, como sí lo fue la Segunda Guerra Mundial, que dejó devastadas a gran parte de las naciones europeas. 


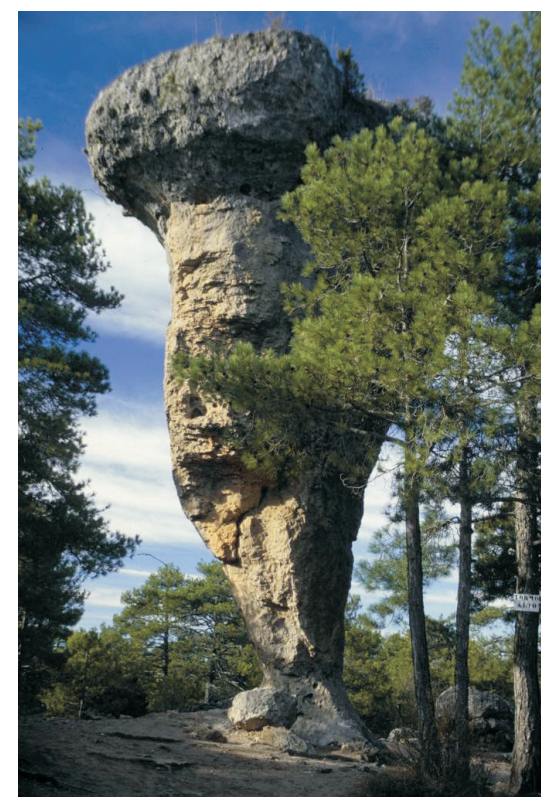

Figura 2 - Ciudad Encantada de Cuenca (España), un relieve kárstico que fue protegido en 1929 con objeto de representar la diversidad geológica española.

En la década de los años 60 y 70 del siglo XX resurgió en Europa una filosofía conservacionista de que la actualmente somos herederos. En muchos países se actualizaron las antiguas leyes de conservación de la naturaleza y se declararon numerosos espacios protegidos. Amparados bajo esas leyes, numerosos lugares de interés geológico fueron protegidos, pero en casi todos lo casos se trataba de leyes que orientaban sus acciones a la preservación de la flora y fauna. La geología y los elementos geológicos perdieron el escaso protagonismo que habían tenido hasta entonces, quedando relegados a un segundo plano y siendo considerados exclusivamente por su aspecto estético o escénico (fig.3). Si bien la conservación de la biodiversidad recibió un importante impulso, el patrimonio geológico, salvo honrosas excepciones como en Gran Bretaña, fue completamente olvidado. Tanto fue así, que los modernos esfuerzos por impulsar la geoconservación en Europa que se han tomado entre los años 90 y la actualidad, en muchos casos han tenido en los propios colectivos y entidades de conservación de la naturaleza su mayor enemigo, pues no entendían que la geología podía formar parte del patrimonio natural. Sea por convencimiento, por la intensa labor de difusión promovida por neocientíficos o por saturación de espacios naturales de flora y fauna, el caso es que en las últimas dos décadas el panorama ha ido cambiando, aunque de manera muy desigual en cada país.

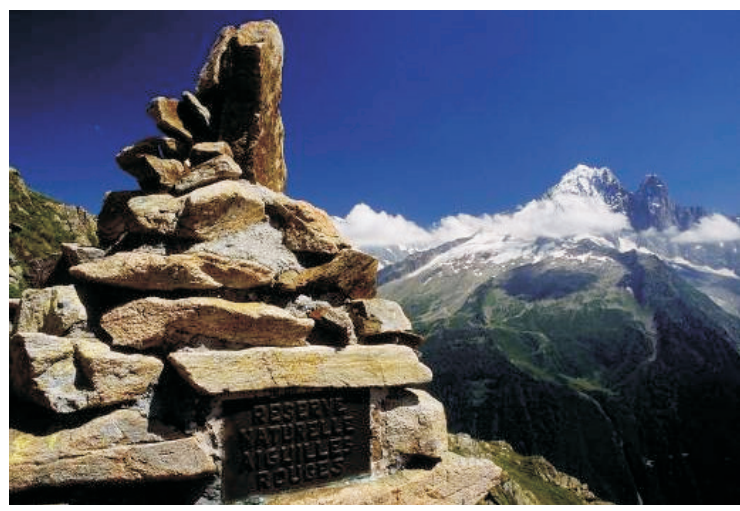

Figura 3 - Reserva Natural de las Aiguilles Rouges (Francia), un espacio protegido declarado en la década de 1970 por su flora y fauna, aunque también incluye elementos geológicos de gran valor.

Hoy en día se considera que la gestión del patrimonio geológico debe basarse en 4 acciones: 1) realizar inventarios, 2) establecer un marco legal que apoye la protección del patrimonio geológico, 3) puesta en marcha de medidas de geoconservación, y 4) divulgación, difusión y promoción de los valores del patrimonio geológico (Carcavilla et al., 2007). Y aquí es donde vienen las mayores diferencias entre los países europeos, pues cada uno de ellos ha afrontado con desigual intensidad y eficacia cada una de estas acciones. Es cierto que prácticamente todos los países cuentan con un inventario, aunque sea preliminar, de lugares de interés geológico. Pero son muy distintas las entidades que los han promovido y, sobre todo, el rango legal que tienen los lugares incluidos en dichos inventarios. El espectro varía entre países con inventarios "informales" promovidos por asociaciones de geocientíficos pero sin ningún apoyo legal, y entre países que cuentan con catálogos aprobados por leyes que otorgan una protección estricta a un número importante de lugares de interés geológico. Por ejemplo, Bulgaria hizo su primer inventario nacional el año 1964 con la identificación de 55 lugares de interés geológico, y países como Gran Bretaña y España dieron sus primeros pasos en la década de los años 70 del siglo XX, aunque en este último caso no llegaría a ser completado por motivos presupuestarios. Por el contrario, otros países han afrontado dichos inventario en épocas muy recientes. El caso es que numerosos países cuentan con inventarios más o menos completos, como Albania que tiene inventariados cerca de 350 lugares de interés geológico (Serjani, 2012), Bulgaria que tiene más de 200 lugares de interés geológico inventariados y cartografiados con criterios uniformes (Todorov, 2012), Dinamarca que cuenta con un inventario de cerca de 400 lugares (Holm, 2012), Eslovaquia con casi 500 (Liscak, 2012) y Rumanía con más de 200 (Andrasanu, 2012). 
Una singularidad de los inventarios realizados por los diferentes países es que en la clasificación de los lugares de interés geológico incluidos se han seguido diferentes sistemas. Si bien en casi todos ellos la clasificación se ha realizado identificando una serie de categorías temáticas, existen muchas diferentes. Así, países con una alta geodiversidad han identificado del orden de 10 categorías de lugares asociadas a disciplinas de Ciencias de la Tierra, como estratigrafía, sedimentología, paleontología, petrología, mineralogía, tectónica, hidrogeología, históricos, cosmogénicos, etc. Este es el caso de los inventarios de países como Rusia, España, Rumanía, Portugal, Italia, Francia o Gran Bretaña. Por el contrario, en otros países geológicamente menos diversos, las clasificaciones son más específicas, como en el caso de Islandia, que define rasgos geomorfológicos del Pleistoceno, elementos del sustrato (volcánicos, estratigráficos, petrológicos, mineralógicos y paleontológicos) y elementos geológicos del Holoceno (Ásbjörnsdóttir et al., 2012) (fig.4); Holanda incluye sobre todo formas del terreno, procesos y sedimentos del Pleistoceno y Holoceno (Ancker \& Jungerius, 2012) y Lituania algo similar, pero vinculados a las glaciaciones

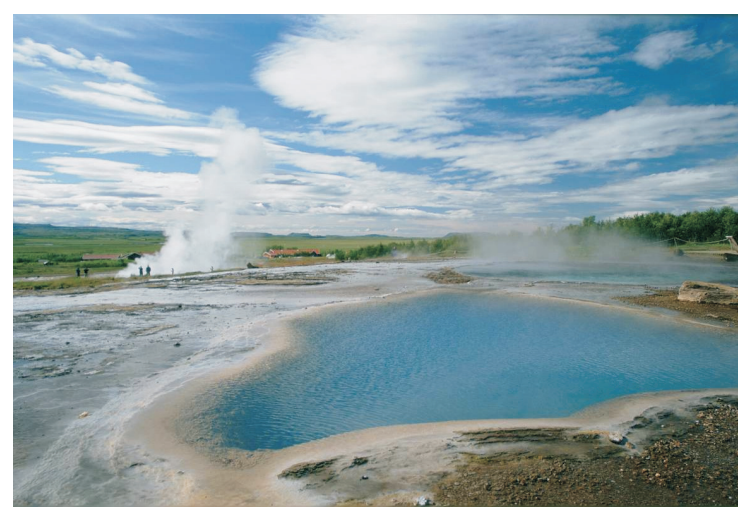

Figura 4 - Zona termal de Geysir (Islandia), sin duda uno de los lugares de Europa con mayor interés geológico debido a la combinación de fenómenos volcánicos y tectónicos activos, unidos al glaciarismo.

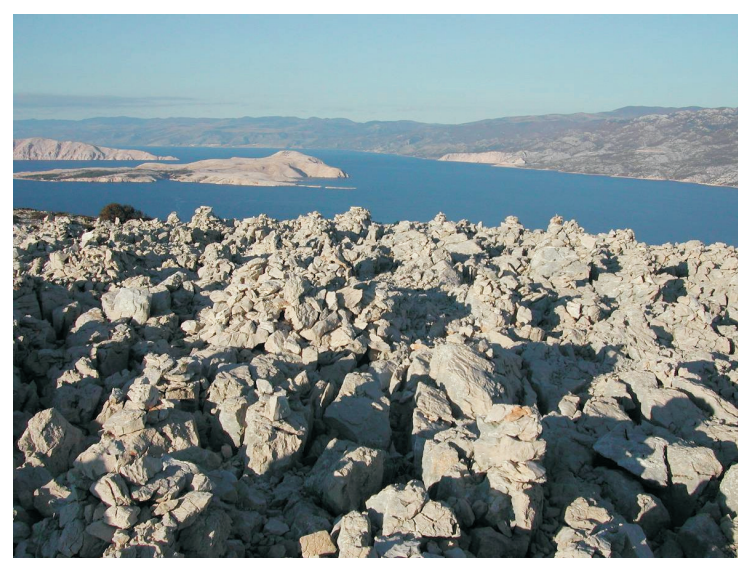

Figura 5 - Isla de Rab (Croacia), con morfologías exokársticas de muy diverso tipo. cuaternarias (Satkunas et al., 2012). Por otro lado, en algunos países, algunos rasgos geológicos adquieren especial protagonismo, por lo que son objeto de una especial atención. Es el caso de los rasgos kársticos, especialmente las cavidades, en Eslovaquia, Eslovenia, Hungría y Croacia (fig.5).

Como ya se ha comentado, la declaración (protección mediante legislación) de espacios protegidos es la principal estrategia utilizada para la protección de elementos geológicos singulares. Este ha sido el mecanismo utilizado en casi todos los países europeos, ya que la protección se refiere a un territorio concreto donde se estipulan una serie de usos permitidos o prohibidos orientados a garantizar la conservación del lugar. Las figuras utilizadas con mayor frecuencia son las de parque nacional, monumento natural, parque natural o reserva natural, con sus consiguientes modalidades nacionales. Pero una característica común en la mayoría de los países europeos es que el hecho de que un elemento geológico esté incluido dentro de un espacio protegido no siempre garantiza su conservación, ya que no siempre los gestores están al corriente de su existencia e importancia. El patrimonio geológico va al remolque de la conservación de la biodiversidad y no ha alcanzado un protagonismo similar. Por otro lado, si se revisan los porcentajes nacionales de territorio protegido en cada país se puede apreciar que hay notables diferencias. Hay naciones como Finlandia que sólo cuentan con un $8 \%$ de su superficie protegida (Kananoja et al., 2012), lo que contrasta con el $42 \%$ de Austria (Hofmann, 2012). En cualquier caso, estos porcentajes sólo aportan una información parcial, ya que la presión sobre el territorio y el porcentaje de suelo ocupado es muy desigual en los diferentes países, así que un índice de protección bajo no es necesariamente sinónimo de una gran presión sobre el medio natural. Una particularidad es que diversos países europeos incluyen en sus territorios nacionales regiones de ultramar, diversificando su red de espacios protegidos de carácter geológico al incluir elementos de contextos geológicos muy diferentes (fig.6).

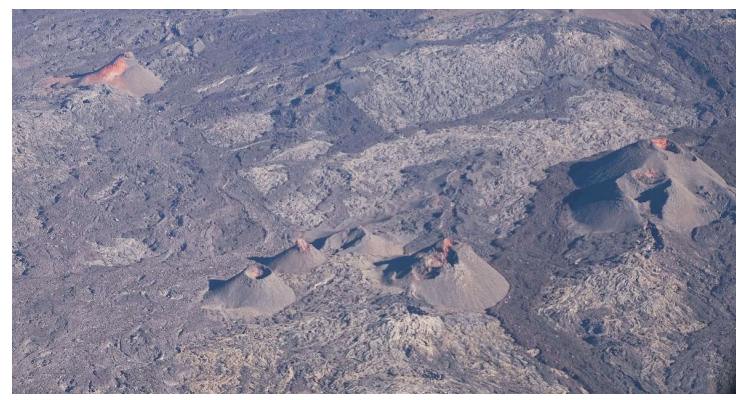

Figura 6 - Conos y coladas recientes en el Piton de la Fournaise, en la Isla Reunión, una de las regiones de ultramar francesas, declarada Parque Nacional y World Heritage. 
Con respecto a la gestión del patrimonio geológico, en muchos de los países europeos hay tres niveles de gobierno: nacional (de todo el país), estatal (de una región o comunidad) y local (de una comarca, provincia o municipio). Generalmente, el gobierno nacional o federal establece una legislación de referencia y luego los gobiernos estatales pueden adaptarla a su territorio manteniéndola como está o volviéndola más restrictiva. Sin embargo, en otros países, la competencia en materia de conservación de la naturaleza no es nacional sino sólo estatal, mediante organismos o agencias específicas. Uno u otro sistema de gestión tienen sus correspondientes ventajas e inconvenientes en relación con la geoconservación, ya que la sensibilidad y predisposición de cada uno de los gobiernos puede ser muy variable. Por otro lado, los servicios geológicos europeos también han mostrado una atención desigual al patrimonio geológico, existiendo ejemplos de alta implicación, como en el caso del Bureau de Recherches Géologiques et Minières (BRGM) francés o el Instituto Geológico y Minero de España (IGME), frente a otros que no han atendido este tema. El papel de los servicios geológicos ha consistido en impulsar inventarios, desarrollar metodologías de inventario, promover normas legales que amparen la geoconservación, potenciar actividades de divulgación y promoción del patrimonio geológico, y participar en programas como el de geoparques.

\section{DOS PROYECTOS EUROPEOS DE DIMENSIÓN INTERNACIONAL: GLOBAL GEOSITES Y GEOPARKS}

Desde diversas naciones europeas se han promovido en las últimas décadas dos iniciativas globales para el conocimiento, conservación y divulgación del patrimonio geológico: los proyectos Global Geosites y Geoparks. Si bien el primero fue abandonado y no se llegó a completar, sí ha servido de estímulo a diversos países para plantear un inventario nacional de lugares de interés geológico de relevancia internacional, como más adelante se describirá. Por su parte, los geoparques surgieron como una iniciativa europea, pero han sobrepasado este ámbito y son una propuesta global, bajo los auspicios de la UNESCO, en clara expansión y con una gran proyección de futuro. A ellos habría que añadir el programa World Heritage de la UNESCO, que incluye entre sus bienes aquellos enclaves que poseen características excepcionales que los convierten en los ejemplos mundiales en su categoría. Bajo esta figura se han declarado elementos geológicos tan singulares como el Volcán Teide (España), la Calzada de los Gigantes (Irlanda) (fig.7), los travertinos de Plitvice (Croacia) o el yacimiento paleontológico de Messel (Alemania).

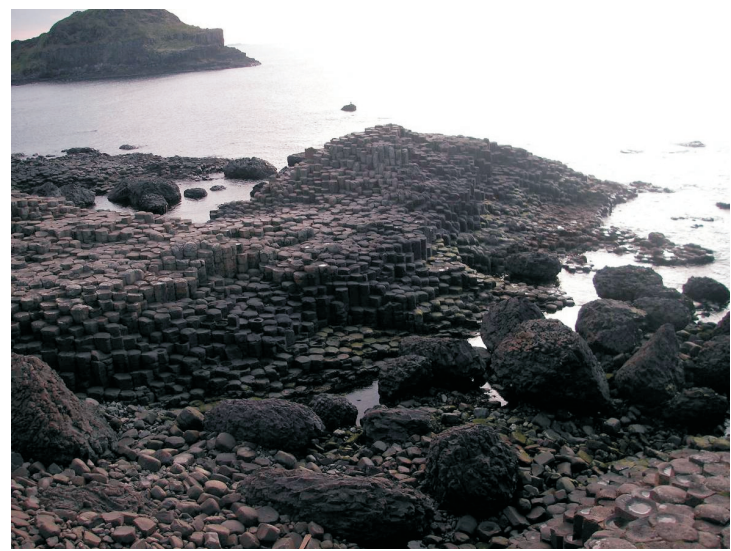

Figura 7 - Calzada de los Gigantes (Irlanda), lugar de interés geológico declarado Patrimonio Mundial por la UNESCO y que recibe al año más de 500.000 visitantes.

El proyecto Global Geosites fue puesto en marcha en 1995 por la Unión Internacional de las Ciencias Geológicas (IUGS) con el co-patrocinio de la UNESCO. Se basaba en la idea de que las estrategias de protección del patrimonio geológico a nivel internacional requieren un inventario previo. Resulta sencillo comprender la dificultad de establecer un inventario mundial de los elementos más sobresalientes del patrimonio geológico del Planeta, teniendo en cuenta la complejidad del registro geológico de la tierra y la gran diversidad de litologías, eventos, paleoambientes, etc, registrados no solo durante los tiempos geológicos, sino también a todo lo largo y ancho del mundo. Por ello, se diseñó una metodología de trabajo que no se dirigía directamente a la selección de los puntos de interés geológico, sino que planteaba un paso previo: elegir en cada país unos contextos geológicos (frameworks) en base a su especial significado en el registro geológico mundial. En una fase posterior, para cada uno de estos contextos geológicos, se seleccionan los puntos de interés más representativos e ilustrativos del mismo. Este concepto de contexto es clave y se refiere a cualquier elemento geológico de escala regional, evento tectónico, metalogenético o de cualquier otra naturaleza, serie estratigráfica, asociación paleobiológica, etc. Los puntos de interés geológico o "geositios" (geosites) no son, por tanto, seleccionados de forma aislada sino en el marco de un contexto previamente escogido por su relevancia internacional. De este modo la selección de los puntos de interés geológico se ve orientada y facilitada por su representatividad dentro del contexto geológico abordado y en comparación con otros en el resto del mundo. El objetivo era seleccionar eventos, áreas y características fundamentales para comprender la historia geológica del Planeta y crear una base de datos asociada a nivel global. No se trata de identificar ejemplos concretos representativos de la geología de un país o lugar, sino contextos geológicos que sean fundamentales para entender la evolución del Planeta, como registros 
sedimentarios, fases tectónicas, sistemas morfogenéticos, etc. Cada país debe identificar sus contextos, para luego comparar con los seleccionados en los países vecinos. Así se seleccionan los contextos de valor supranacional y global. Siguiendo esta metodología, diversos países realizaron sus listados, como Polonia, Bulgaria, España, Reino Unido y Portugal. Como se ha mencionado anteriormente, el proyecto a escala global fue abandonado, pero esta metodología sirvió a diversos países para realizar un listado homogéneo de los lugares de interés geológico de relevancia internacional contenidos en su territorio, constituyendo una valiosa herramienta para la realización de inventarios nacionales. Actualmente, ProGEO ha vuelto sobre la idea y ha empezado a promover la continuación del proyecto y que éste sea retomado por la IUGS; habiendo conseguido ya que la Unión Internacional para la Conservación de la Naturaleza (UICN) lo adopte en una resolución (Res 5.048 de 2012).

Por su parte, los Geoparques surgieron a principios de la década de los 90 en Europa, siendo Francia, Alemania, Grecia y España los socios fundadores (fig.8). Desde entonces, su número ha ido en aumento y ya es una iniciativa global. Un geoparque (Geopark o parque geológico) es un territorio que presenta un patrimonio geológico notable y que lleva a cabo un proyecto de desarrollo basado en su promoción turística, de manera que debe tener unos objetivos económicos y de desarrollo claros. La declaración de un geoparque se basa en tres principios: 1) la existencia de un patrimonio geológico que sirva de protagonista y eje conductor, 2) la puesta en marcha de iniciativas de geoconservación y divulgación, y 3) favorecer el desarrollo socioeconómico y cultural a escala local. Así que tres son los pilares que sustentan la creación y funcionamiento de un geoparque: patrimonio geológico, geoconservación y desarrollo local. Por ello, los geoparques deben tener unos límites claramente definidos y una extensión adecuada para asegurar el desarrollo económico de la zona, pudiendo incluir áreas terrestres, marítimas o subterráneas. En Europa existen ya más de 50 geoparques en casi una veintena de países. Italia, España y Reino Unido son los países que por el momento más geoparques aportan a la Red Europea. En estos países, los geoparques se han afianzado como iniciativas para promover el uso y conservación del patrimonio geológico. Es importante dejar claro que los geoparques no son espacios naturales protegidos en sentido estricto (aunque pueden coincidir en espacio y tiempo), sino un territorio con una estrategia de gestión orientada a los tres principios indicados anteriormente.

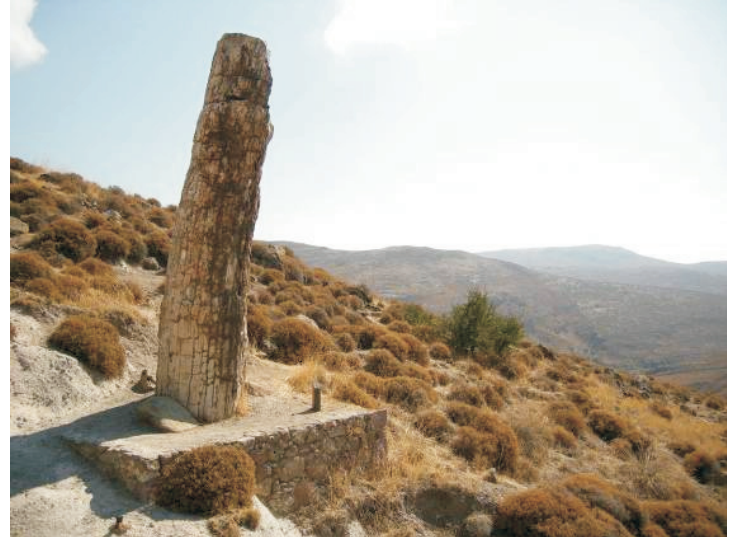

Figura 8 - Bosque Petrificado de Lesvos (Grecia), uno de los cuatro lugares fundadores de la iniciativa geoparques a finales de los años 90.

\section{ALGUNOS EJEMPLOS SINGULARES}

Dentro del contexto europeo, hay muchos ejemplos de buenas prácticas relacionadas con el patrimonio geológico y la geoconservación. Pero entre todas ellas se pueden elegir unos ejemplos que muestran enfoques particulares a una misma problemática y que han mostrado ser eficaces, y pueden servir de ejemplo o estímulo a otros países.

\subsection{Inventarios nacionales: Reino Unido}

En relación con los inventarios, destaca el caso del Reino Unido, donde el estudio del patrimonio geológico se inició en 1949, aunque el estudio sistemático en realidad comenzó en 1977 y se dio por concluido en 1990. Este inventario poseía una clara vocación conservacionista, ya que estaba acompañado de un mecanismo legal de protección de los LIGs. Esto último es lo que da un especial valor a dicho inventario, y la prueba es que, a pesar del paso del tiempo, existen en la actualidad pocos inventarios europeos que contemplen dicho aspecto.

En este contexto están los Geological Conservation Review sites de Gran Bretaña (GCR sites). Estos lugares de interés fueron descritos dentro del marco del proyecto Geological Conservation Review, que consistió en un inventario nacional de lugares de interés geológico que inició su búsqueda de lugares en 1977 y finalizó en 1990, auspiciado por el Joint Nature Conservation Committee (JNCC). No obstante, las labores de selección de enclaves puntuales ya se habían iniciado a finales de la década de los 40 . Dentro de este proyecto se incluía un ambicioso plan de documentación sobre la geología del país y la consulta a numerosos especialistas de diferentes especialidades geológicas. El resultado fue la publicación de 44 volúmenes temáticos de la geología del Reino Unido, denominados Geological Conservation Review Series (Ellis, 2005). 
El equivalente en Irlanda del Norte se denominó Earth Science Conservation Review (ESCR).

De los más de tres mil GCR sites identificados se seleccionaron los más importantes (por su relevancia internacional y nacional) para que gozaran de una protección legal (Joint Nature Conservation Committee, 2005). De esta manera surgieron los cerca de 1.400 Sites of Special Scientific Interest (SSSI) que constituyen una red de lugares importantes para el "pasado, presente y futuro desarrollo de la geología en Reino Unido", de manera que representan lo más significativo de la geología de ese país, reflejando la diversidad de su historia geológica. Su declaración se basa en la Wildlife and Countryside Act de 1981, aunque recientemente en el año 2000 una nueva normativa denominada Countryside and Rights of Way Act 2000 (CROW Act) actualizó y aumentó el régimen de protección. Es muy importante recalcar que los SSSI gozan de un sistema de protección legal y un amplio reconocimiento a escala nacional, lo que hace que la conservación del patrimonio geológico en Gran Bretaña sea de las más avanzadas de Europa. La equivalencia en Irlanda del Norte de los SSSI se denominan Areas of Scientific Interest (ASSI), y en Gales se denominan Site of Importance for Nature Conservation (SINC).

Otro ejemplo de la manera de trabajar en el inventario y conservación del patrimonio geológico en Reino Unido es el de los Regionally Important Geological/Geomorphological Sites (RIGS). También fueron establecidos en Gran Bretaña en 1990 por el Nature Conservancy Council (NCC) y consiste en un programa desarrollado por iniciativas voluntarias de asociaciones locales no gubernamentales (RIGS groups) que cuentan con la aprobación informal de las autoridades locales. Frente a los SSSI y los GCR sites, que son propuestas institucionales, los RIGS son iniciativas voluntarias de carácter particular que no poseen ningún reconocimiento legal oficial. Los RIGS groups definen los County Geological Sites (CGS), también Ilamados RIGS sites cuando el propietario del terreno permite el acceso. Ambos sirven de complemento a los SSSI y son puestos en conocimiento de las autoridades y tenidos en cuenta en la planificación. En la actualidad existen más de 50 RIGS Groups en Reino Unido, formados mayoritariamente por museos y asociaciones ecologistas, y en menor medida, por sociedades geológicas y universidades.

\subsection{Inventario nacional e internacional en el marco del proyecto Global Geosites: Portugal}

Algunos países han desarrollado la metodología del proyecto Global Geosites como sistema para iniciar o completar su inventario nacional. Dos de los casos más sobresalientes son los de Portugal y España, que incluso han colaborado para aplicar una metodología común que les permita complementar la información de cada país.
En el caso de España, el proyecto se desarrolló entre 1999 y 2008 liderado por el IGME, que hace las veces de Servicio Geológico Nacional. En una primera fase se identificaron 20 contextos geológicos españoles de relevancia internacional (frameworks) que fueron presentados en el 31 Congreso Geológico Internacional de Río de Janeiro en el año 2000. A partir de entonces, se empezaron a definir los lugares de interés geológico que representaban esos contextos, que fueron 148 repartidos en 216 recintos. Actualmente España cuenta con una cartografía precisa de esos enclaves y con una base de datos de los lugares de interés geológico que, como más adelante se explicará, tienen reflejo en el marco legal de conservación del patrimonio natural nacional.

Portugal inició las labores del proyecto Global Geosites más tarde que España. Pero supo aprovechar la experiencia de su país vecino para identificar sus contextos de una manera rápida y eficaz. En el año 2000 se creó el grupo portugués de ProGeo y en el año 2004 se propuso una primera lista con 14 contextos de relevancia internacional (Brilha et al., 2005), varios de los cuales tenían una clara conexión con contextos definidos en España. Posteriormente, fueron definidos otros contextos con la idea de representar la geodiversidad portuguesa, de manera que se definieron un total de 29 contextos y 326 lugares de interés geológico. Algunos de los contextos definidos previamente fueron redefinidos y/o englobados en nuevas denominaciones, más amplias y que daban cabida a otros lugares de interés relacionados (Brilha et al., 2009). En ellos se ha estimado su valor intrínseco y su vulnerabilidad para orientar su gestión a las autoridades competentes. De esta manera, la metodología del proyecto Global Geosites ha sido utilizada en Portugal para definir 15 contextos de relevancia internacional y otros 14 de relevancia nacional o regional, completando de una manera muy eficaz un inventario nacional que combina la singularidad con la representatividad.

\subsection{Legislación para la geoconservación: el caso de España}

Para que la conservación del patrimonio geológico sea realmente eficaz es necesario contar con un marco legal que ampare su preservación y protección. En la mayoría de los países europeos la protección se realiza en el marco de leyes de conservación del patrimonio natural, definiendo figuras de protección que pueden englobar o proteger elementos geológicos, como parques nacionales o naturales, monumentos naturales, etc. En algunos países la legislación para la conservación del patrimonio natural pone especial atención en el patrimonio geológico, como ocurre en el caso de España.

Durante el año 2007, tres nuevas leyes supusieron un cambio radical en lo referente a la legislación para la geoconservación en España. 
La primera de estas leyes fue la Ley 42/2007 de Patrimonio Natural y Biodiversidad, en la que tuvieron una activa participación el IGME, el Colegio Profesional de Geólogos (ICOG) y la Sociedad Geológica de España (SGE). La receptividad del Ministerio de Medio Ambiente tuvo como resultado que, desde primera vez desde los comienzos de la geoconservación en España, una Ley tuviera entre sus principios inspiradores la conservación de la geodiversidad. Las principales aportaciones de la Ley son de tres tipos: 1) menciones expresas en sus artículos a la geología, patrimonio geológico, geoparques y geodiversidad; 2) crea un inventario nacional de patrimonio natural que tendrá un inventario de lugares de interés geológico; 3 ) adopta medidas de conservación del patrimonio geológico y la geodiversidad; y 4) amplia los bienes que pueden ser protegidos, haciendo mención concreta a los elementos geológicos como parte de los monumentos, parques y reservas naturales.

Además, esta Ley dice explícitamente que el Ministerio de Medio Ambiente, con la colaboración de las comunidades autónomas y de las instituciones y organizaciones de carácter científico, elaborará un Inventario Español del Patrimonio Natural y de la Biodiversidad, que incluirá un inventario de Lugares de Interés Geológico representativo de, al menos, las unidades y contextos geológicos (frameworks) del proyecto Global Geosites y otros ocho sistemas geológicos representativos de la geodiversidad española (fig.9). También estipula que se llevará a cabo un Plan Estratégico Estatal del Patrimonio Natural y de la Biodiversidad que incluirá, a su vez, un diagnóstico anual de la situación y de la evolución del patrimonio natural y la biodiversidad y geodiversidad.

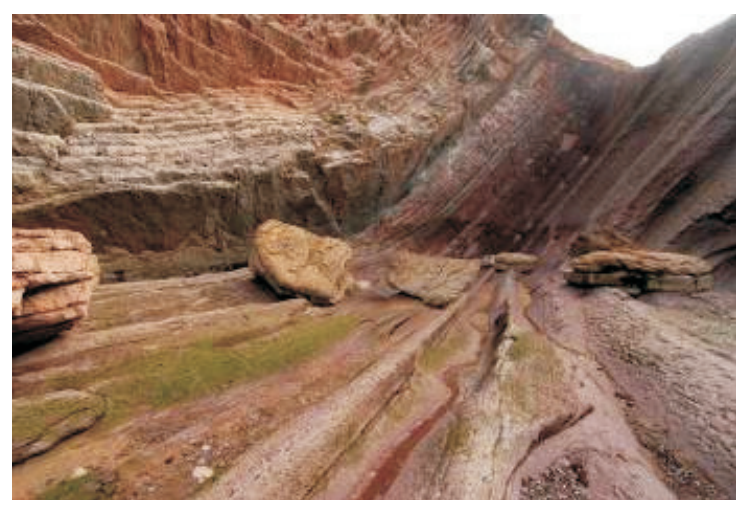

Figura 9 - Límite Cretácico-Terciario en Zumaya (España), uno de los lugares de interés geológico de relevancia internacional identificados en el proyecto Global Geosites.

La segunda ley importante para la geoconservación en España es la Ley de Parques Nacionales, que incluye en un anexo los sistemas naturales españoles que deben estar representados en la Red de Parques Nacionales, entre los que se encuentra una decena de sistemas geológicos. Por su parte, la Ley (45/2007) para el Desarrollo Sostenible en el Medio Rural menciona de manera explícita el turismo geológico y minero (geoturismo) y propone aprovechar los recursos geológicos para potenciar el desarrollo sostenible en el medio rural, dando prioridad a la conservación del medio ambiente, el paisaje y el patrimonio natural y cultural, algo que va en clara sintonía con la declaración de Geoparques, de los cuales existen 9 en España (año 2013) (fig.10).

Como se puede ver, el nuevo marco normativo pone solución (teóricamente) a algunos de los problemas esenciales de la geoconservación en España, como eran la ausencia de un marco legal adecuado, la ausencia de inventario o la inadecuada definición de las figuras de protección. Los retos para los próximos años serán aprovechar la oportunidad que ofrecen estas nuevas leyes y seguir implantando los programas internacionales (fundamentalmente Geoparks y Global Geosites) para, desde la perspectiva nacional y la internacional, dar el impulso definitivo a la geoconservación en España.

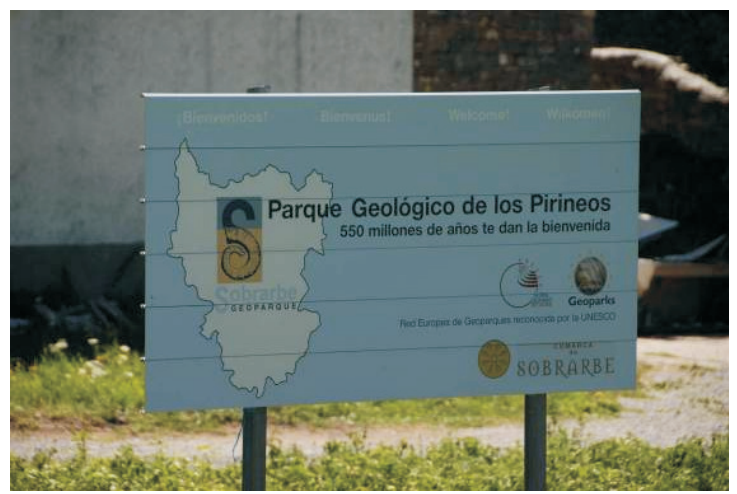

Figura 10 - Geoparque de Sobrarbe (España), uno de los más de 50 pertenecientes a la Red Europea de Geoparques.

\subsection{La geoconservación integrada en un marco holístico: Noruega}

Noruega es un país europeo pero no pertenece a la Unión Europea. Como algunos otros países, recientemente ha promulgado leyes relacionadas con la conservación de la naturaleza incorporando referencias a la geoconservación. La Ley de Diversidad Natural del año 2009 establece como uno de sus principios proteger la diversidad biológica y geológica a través de la conservación y el uso sostenible.

Ya se mencionó con anterioridad que la geoconservación tiene raíces muy antiguas en Noruega, con una primera ley de conservación de la naturaleza promulgada en 1910 (Erikstad, 2012). Aunque el patrimonio geológico no era mencionado de manera específica como justificación para la adopción de medidas de conservación en la legislación, la realidad es que sí ha formado parte del sistema de protección noruego entre 1954 y 2009. Especialmente en el periodo comprendido entre 1970 y la década de los 90, cuando fueron realizados numerosos inventarios y fueron 
declarados numerosas áreas protegidas en forma de reservas, monumentos y paisajes naturales protegidos. Con posteridad, la geología quedó relegada a un segundo plano, como ocurrió en muchos otros países europeos, quedando la geoconservación eclipsada por la protección de la biodiversidad. Sin embargo, la nueva legislación ofrece numerosas posibilidades a la geoconservación y la integra dentro de la gestión natural.

Noruega contaba con 2.759 áreas protegidas en enero de 2012, cubriendo un $17 \%$ de su superficie. Aproximadamente 200 de ellas están etiquetadas como áreas de conservación geológica, ya que en ellas dominan los aspectos geológicos o la conservación geológica está integrada en su plan de gestión. Muchas de ellas son realmente pequeñas, pero las de gran tamaño (como parques nacionales o paisajes protegidos) contienen valores geológicos de gran extensión integrados en el paisaje. Una clara tendencia dentro de los parques nacionales es incluir la conservación de los elementos geológicos entre sus objetivos, y aún en los casos donde no se refleja de manera expresa, está protegido desde una perspectiva general como elemento constitutivo del paisaje y de la naturaleza.

Las islas árticas de Svalvard tienen su propia legislación medioambiental, estando protegido un $60 \%$ de su territorio.

La amplitud de miras de la nueva legislación, que incluye la conservación y el uso sostenible como principios, proporciona la oportunidad de incluir el patrimonio geológico y la geodiversidad en la gestión ambiental. Además, ofrece oportunidades para la geoconservación al margen de la declaración de áreas protegidas, integrándola en la planificación territorial, evaluaciones de impacto ambiental y estrategias del paisaje. También incluye elementos geológicos dentro de los sistemas naturales del país, que incluyen hábitats y paisajes, y empiezan a incorporarse en los inventarios locales y en la lista nacional de sistemas naturales amenazados (Erikstad, in press).

La geoconservación en Noruega aún está por detrás de la preservación ecológica, ambiental y del patrimonio cultural, pero esta legislación de enfoque holístico puede favorecer y beneficiar a la biodiversidad, a la geodiversidad y al paisaje.

\section{DIRECTIVAS COMUNITARIAS COMO APOYO A LA GEOCONSERVACIÓN}

Los países de la Unión Europea deben sumar a su marco normativo nacional una serie de recomendaciones y directivas de ámbito europeo, siendo optativo en las primeras de ellas y obligatorio en las segundas traspasarlas a su legislación. En la actualidad no existe ninguna directiva europea referida al patrimonio geológico, pero sí hay varias que pueden ayudar o suponer un apoyo a la geoconservación.
En el año 2004 el Consejo de Ministros de la Unión Europea (Council of Europe, 2004) aprobó las recomendaciones para la conservación del patrimonio geológico y de áreas de interés geológico (Rec, 2004). Entre estas recomendaciones se encontraban las de promover y realizar inventarios, reforzar los mecanismos legales para proteger áreas de interés geológico o asignar recursos presupuestarios para financiar las iniciativas expuestas anteriormente. A pesar de que suponían un excelente marco para el impulso de políticas nacionales de geoconservación, al ser únicamente recomendaciones, los diferentes países las aplicaron de manera muy desigual y no hubo un seguimiento tras el plazo de 5 años que establecía. Por desgracia, estas recomendaciones no vinieron seguidas de una directiva de geoconservación, que habría sido la herramienta de impulsarla definitivamente en la Unión.

Por su parte, en el año 2000 fue adoptada la Convención Europea del Paisaje, que fue puesta en marcha en 2004. Se trata de la primera convención que aborda el multidisciplinar asunto del paisaje con el objetivo de promover su protección. Parte de la idea de que cada paisaje está formado por diferentes componentes y estructuras, entre las cuales la geología

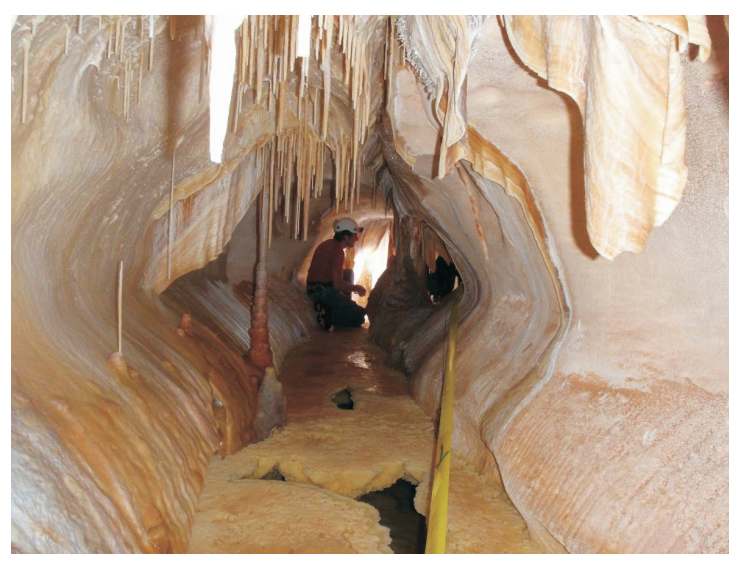

Figura 11 - Algunos de los hábitats incluidos en la Directiva Hábitats de la red Natura 2000 de la Unión Europea tienen una clara componente geológica, como las cavidades kársticas, por lo que muchas de ellas gozan de una protección al menos indirecta. Cueva de Vallgornera (España).

es una de ellas. Por ello, aunque la directiva no centra su atención en el patrimonio geológico, puede servir como herramienta para la geoconservación de aquellos elementos geológicos que formen parte importante de paisajes singulares y/o representativos. Algo parecido ocurre con la Directiva de Hábitats, creada con objeto de proteger los hábitats más singulares de la Unión Europea. Aunque esta directiva está diseñada con un marcado carácter fitosociológico, varios de los hábitats incluidos en su listado como de protección especial son elementos geológicos, como tobas calcáreas, cavidades o sistemas de dunas (fig.11). Por ello, puede suponer una interesante herramienta para promover la geoconservación en esos sistemas. Su adopción como 
una directiva sería una gran paso hacia delante, aunque el esfuerzo de reconocer la geoconservación con su propio derecho dentro del sistema de conservación de la naturaleza (la directiva de hábitats y la red NATURA 2000) debe ser establecido en el trabajo práctico del día a día (Erikstad, en prensa).

Como se ha comentado con anterioridad, en Europa la legislación y la forma de actuar de cada uno de los países en materia de geoconservación es muy desigual (Wimbledon \& Smith-Meyer, 2012; Erikstad, 2008). La perspectiva internacional no proporciona grandes ventajas porque la geoconservación no está contemplada en ninguna directiva de la Unión Europea, de manera que no existe ninguna imposición para afrontarla. Se propuso su integración como parte de la Estrategia Europea del Suelo, que aspira a convertirse en una directiva. En ella, el patrimonio geológico sería integrado como una de las funciones del suelo a proteger, entendido el suelo en su sentido más amplio ya que puede contener o constituir ese tipo de patrimonio. En caso de que fuera aceptada, constituiría un importante paso adelante, aunque dicha directiva ha sido retrasada (o incluso detenida) por los numerosos desacuerdos entre los países miembros. El resultado es que la geoconservación no goza del mismo reconocimiento que la conservación de otros elementos del patrimonio natural.

Por otro lado, en Europa se definió la primera convención internacional dedicada exclusivamente al paisaje. Se integra dentro del programa de Medio Ambiente del Consejo de Europa y Naciones Unidas denominado "Estrategia pan-europea para la diversidad biológica y del paisaje", que fue aprobada por los ministros de medio ambiente de los 55 países implicados en Sofía (Bulgaria) en 1995. La acción 4 del Plan de Acción para 1996-2000 se denominó "Conservación de los paisajes" y entre los objetivos formulados para ser alcanzados en el año 2000 figuraba "prevenir futuros deterioros de los paisajes y del patrimonio geológico y cultural a ellos asociados y preservar su belleza e identidad. Corregir la ausencia de percepción integral de los paisajes como un mosaico de rasgos culturales, naturales y geológicos y establecer una mayor conciencia pública y por parte de las administraciones y mejorar su protección en toda Europa" (Council of Europe, 2006). La convención del paisaje aporta una visión muy amplia englobando todos los elementos que le confieren calidad y singularidad, por lo que puede ser una buena herramienta para la geoconservación en Europa. Además, hace especial énfasis en cómo la gente percibe el paisaje y en la importancia de la participación e implicación local.

\section{EL PAPEL DE PROGEO}

ProGEO es la Asociación Europea para la Conservación del Patrimonio Geológico. Se trata de una asociación abierta a todo tipo de profesionales e instituciones que trabajan hacia ese objetivo, y cuenta con miembros de la mayoría de las naciones europeas. ProGEO trabaja para promover la protección de los paisajes y lugares de interés geológico representativos y más importantes de Europa, así como las múltiples formas en que se muestran los fenómenos geológicos y sus resultados, analizando su valor y destacando sobre todo su interés científico e importancia cultural para definirlo como patrimonio y que sea protegido como tal. ProGEO intenta dar a la conservación del patrimonio geológico y la geodiversidad una voz más fuerte en Europa, y servir como foro de discusión sobre estos temas, asesorando y tratando de influenciar en las políticas y la administración del patrimonio (Fig.12).

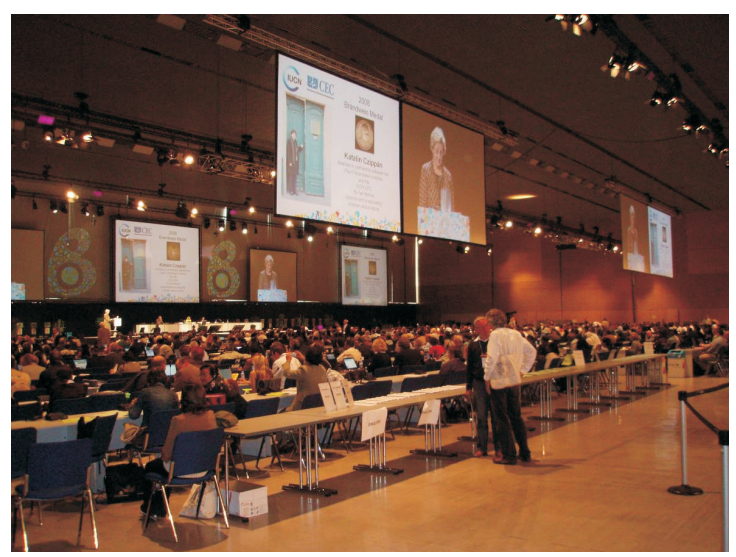

Figura 12 - ProGEO participa activamente en la integración de la geoconservación en diferentes foros, por ejemplo asistiendo a las dos últimas reuniones de la Unión Internacional de Conservación de la Naturaleza (UICN) en 2008 y 2012.

ProGEO es un marco de referencia actual a nivel no solo europeo sino también internacional para la conservación y estudio del patrimonio geológico. El objetivo principal de esta asociación es dar a la geoconservación un mayor protagonismo y relevancia, para que sea tenida en cuenta en las decisiones sobre conservación del patrimonio natural. ProGEO organiza reuniones periódicas y trabaja mediante subcomisiones regionales que agrupan países por criterios geográficos y culturales. La web actual es: http://www.progeo.se

\section{PERSPECTIVAS DE FUTURO}

Como se ha visto con anterioridad, no existe una política común para abordar la valoración, estudio y conservación del patrimonio geológico en Europa. Los diferentes países han abordado el tema según la receptividad de las administraciones y el dinamismo de los propios geólogos, dando como resultado diversos modelos que tienen sus particularidades. A pesar de que se asume que el mayor campo de acción para la geoconservación se sitúa en el ámbito local, sin el impulso de leyes nacionales o directivas transnacionales 
es difícil que se alcancen objetivos realmente ambiciosos. Actualmente, y a pesar de que se han hecho muchos avances para el diseño de directivas europeas, la geoconservación está en un claro segundo plano frente a la conservación de la biodiversidad. La promulgación de leyes específicas para la conservación del patrimonio geológico solucionaría este asunto, pero es poco probable que las administraciones redacten este tipo de normas legales. Por ello, son también muy interesantes las normas legales de carácter holístico, que pueden constituir una buena aproximación porque permiten vincular al patrimonio geológico con otros elementos del medio natural.

Por otro lado, es interesante cómo, a falta de directivas europeas, diferentes instituciones con ProGEO a la cabeza promueven trabajos trasnacionales, como retomar las labores de inventario del proyecto Global Geosites en aquellos lugares donde los trabajos nacionales están más avanzados, como Europa suroccidental o la región báltica. Por su parte, los geoparques, que van en aumento, también juegan un importante papel, sobre todo para mostrar cómo el patrimonio geológico puede constituir un importante motor para el desarrollo de la economía local. Sin duda, la concienciación y la educación son las armas para el futuro, por lo que la divulgación no sólo es un recurso interesante, sino una garantía para la geoconservación a largo plazo. Mientras tanto, es esencial trabajar en elaborar inventarios, redactar leyes y asegurar la preservación de los lugares de interés geológico más amenazados.

\section{Referencias}

ALEXANDROWICZ, Z. 2012. Poland. In: WIMBLEDON, W.A.P. \& SMITH-MEYERS, S. (eds) Geoheritage in Europe and its conservation. ProGEO, Oslo, 254-263.

ANCKER, J.A.M. \& JUNGERIUS, P.D. 2012. The Netherlands. In: WIMBLEDON, W.A.P. \& SMITHMEYERS, S. (eds) Geoheritage in Europe and its conservation. ProGEO, Oslo, 246-253.

ANDRASANU, A. Romania. In: WIMBLEDON, W.A.P. \& SMITH-MEYERS, S. (eds) Geoheritage in Europe and its conservation. ProGEO, Oslo, 274-287.

ÁSBJÖRNSDÓTTIR, L., EINARSSON, S \& JÓNASSON, K. 2012. Iceland. In: WIMBLEDON, W.A.P. \& SMITHMEYERS, S. (eds) Geoheritage in Europe and its conservation. ProGEO, Oslo, 170-179.

BRILHA J., BARRIGA F., CACHAO M, COUTO M.H., DIAS R., HENRIQUES M.H., KULLBERG J.C., MEDINA J., MOURA D., NUNES J.C., PEREIRA D., PEREIRA P., PRADA S. \& SÁA. 2009. Implementaçao de contextos geológicos para a inventariaçao do património geológico Portugues. Resúmenes, VIII Reunión de la Comisión de Patrimonio Geológico de la Sociedad Geológica de España, Daroca (Zaragoza), June 18-21, 11.

BRILHA, J., ANDRADE, C., AZERÊDO, A., BARRIGA, F.J.A.S., CACHÃO, M., COUTO, H., CUNHA, P.P., CRISPIM, J.A., DANTAS, P., DUARTE, L.V., FREITAS, M.C., GRANJA, H.M., HENRIQUES, M.H., HENRIQUES, P., LOPES, L., MADEIRA, J., MATOS, J.M.X., NORONHA, F., PAIS, J., PIÇARRA, J., RAMALHO, M.M., RELVAS, J.M.R.S., RIBEIRO, A., SANTOS, A. , SANTOS, V.F. \& TERRINHA, P. 2005. Definition of the Portuguese frameworks with international relevance as an input for the European geological heritage characterisation. Episodes, Vol. 28, no. 3, 177-156

CARCAVILLA, L., DURÁN, J.J., GARCÍA-CORTÉS, A. \& LÓPEZ-MARTÍNEZ, J. 2009. Geological heritage and geoconservation in Spain: past, present and future. Geoheritage, Volume 1, Issue 2, 75-91.
CARCAVILLA, L., LÓPEZ-MARTÍNEZ, J. Y DURÁN, J.J. 2007. Patrimonio geológico y geodiversidad: investigación, conservación, gestión y relación con los espacios naturales protegidos. Instituto Geológico y Minero de España. Serie Cuadernos del Museo Geominero, no 7. Madrid. $360 \mathrm{p}$.

COUNCIL OF EUROPE. 2006. Landscape and sustainable development: challenges of the European Landscape Convention. Strasbourg (France): Council of Europe.

ELLIS, N. 2005. The Geological Conservation Review in the 21st century. Abstracts del IV International Symposium ProGEO on the Conservation of the Geological Heritage, 11. University of Minho. Braga.

ERIKSTAD, L. 2008. History of geoconservation in Europa. In: BUREK, C.V. \& PROSSER, C.D. (eds.) The history of geoconservation. 300. Geological Society, London, 249-256.

ERIKSTAD, L. 2012. Norway. In: WIMBLEDON, W.A.P. \& SMITH-MEYERS, S. (eds) Geoheritage in Europe and its conservation. ProGEO, Oslo, 246-253.

ERIKSTAD, L. in press. Geoheritage and geodiversity management - the questions for tomorrow. Proc. Geol. Assoc. In press. Available online 4 August 2012, http://dx.doi.org/10.1016/j.pgeola.2012.07.003)

HLAD, B. 2012. Slovenia. In: WIMBLEDON, W.A.P. \& SMITH-MEYERS, S. (eds) Geoheritage in Europe and its conservation. ProGEO, Oslo, 320-333.

HOFMANN, T. 2012. Austria. In: WIMBLEDON, W.A.P. \& SMITH-MEYERS, S. (eds) Geoheritage in Europe and its conservation. ProGEO, Oslo, 30-39.

HOLM, L. 2012. Denmark. In: WIMBLEDON, W.A.P. \& SMITH-MEYERS, S. (eds) Geoheritage in Europe and its conservation. ProGEO, Oslo, 100-105.

JACOBS, P. 2012. Austria. In: WIMBLEDON, W.A.P. \& SMITH-MEYERS, S. (eds) Geoheritage in Europe and its conservation. ProGEO, Oslo, 52-61. 
JOINT NATURE CONSERVATION COMMITTEE. 2005. Geological Conservation Review sites. Disponível em: http://www.jncc.gov.uk/earthheritage/gcrdb/defaul t.htm. Acessado em 9 outubro 2005.

KANANOJA, T., SUOMINEN, V \& NEROMEN K. 2012. Finland. In: WIMBLEDON, W.A.P. \& SMITH-MEYERS, S. (eds) Geoheritage in Europe and its conservation. ProGEO, Oslo, 115-123.

LISCAK, P. 2012. Slovakia. In: WIMBLEDON, W.A.P. \& SMITH-MEYERS, S. (eds) Geoheritage in Europe and its conservation. ProGEO, Oslo, 310-121.

MALKOWSKI, S. 1928. Buts et signiication de la protection des monumnets de la nature inanimée. Monuments de la Naturre Inanimée de la Republique Polonaise, 1, 5-9.

MARTINI, G. (coord.). 1994. Actes du premier symposium international du patrimoine géologique, Digne-les-Bains, 11-16 juin 1991, Mémoires de la Société Géologique de France, $n^{\circ} 165,276 \mathrm{p}$.

RÖHLING, H.G. 2012. Germany. In: WIMBLEDON, W.A.P. \& SMITH-MEYERS, S. (eds) Geoheritage in Europe and its conservation. ProGEO, Oslo, 132-143.

SATKUNAS, J., LINCIUS, A \& MIKULENAS, V. 2012. Lithuania. In: WIMBLEDON, W.A.P. \& SMITH-MEYERS, $S$. (eds) Geoheritage in Europe and its conservation. ProGEO, Oslo, 216-223.

SERJANI, A. 2012. Albania. In: WIMBLEDON, W.A.P. \& SMITH-MEYERS, S. (eds) Geoheritage in Europe and its conservation. ProGEO, Oslo, 20-29.

TODOROV, T. Bulgaria. In: WIMBLEDON, W.A.P. \& SMITHMEYERS, S. (eds) Geoheritage in Europe and its conservation. ProGEO, Oslo, 68-79.

WIMBLEDON, W.A.P. Preface. In: WIMBLEDON, W.A.P. \& SMITH-MEYERS, S. (eds) Geoheritage in Europe and its conservation. ProGEO, Oslo, 6-19.

COUNCIL OF EUROPE. 2004. Recommendation $\operatorname{Rec}(2004) 3$ on conservation of the geological heritage and areas of special geological interest. D i s p o n íve l e $\mathrm{m}$ : https://wcd.coe.int/ViewDoc.jsp?id=740629. Acessado em 10 febrero 2013. 